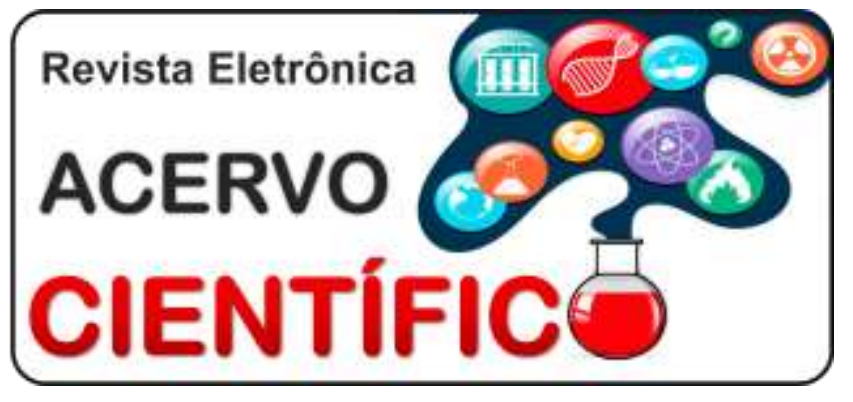

\section{ARTIGO ORIGINAL}

Recebido em: 10/2019

Aceito em: $11 / 2019$

Publicado em: 1/2020

\title{
Avaliação do controle das medidas de biossegurança adotadas por acadêmicos de Odontologia
}

\author{
Evaluation of the control of biosafety measures adopted by dental students
}

Evaluación del control de las medidas de bioseguridad adoptadas por estudiantes de odontología

Téssia Richelly Nóbrega Borja de Melo ${ }^{1 *}$, Poliana de Santana Costa ${ }^{1}$, Vanessa da Silva Oliveira ${ }^{1}$, Matheus de Almeida Germano Diniz ${ }^{1}$, Augusto Garcia de Oliveira Júnior ${ }^{1}$.

Resumo: Este artigo buscou verificar o conhecimento de acadêmicos de odontologia sobre as normas de biossegurança. Tratou-se de um estudo descritivo, através da utilização de questionário. A amostra foi comporta por acadêmicos concluintes do curso de odontologia $(n=30)$. Os dados foram tabulados no programa SPSS (Statistical Package for Social Sciences). 100\% da amostra faz a proteção ao paciente; $68 \%$ utiliza os óculos de proteção; $96 \%$ descarta materiais cortantes em reservatório adequado; $89 \%$ utiliza luva de borracha para lavar instrumentais; $96 \%$ utiliza álcool à $70 \%$ como desinfetante; $82 \%$ relatou "bom" conhecimento de biossegurança, e a existência de protocolos para profissionais acidentados; $83 \%$ evita "tocar objetos com as mãos enluvadas"; e $68 \%$ fazem a lavagem das mãos antes e depois de usar luvas; $94 \%$ realiza esterilização de brocas. A maioria dos acadêmicos conhecem os protocolos de biossegurança e executam os procedimentos clínicos de acordo com as normas estabelecidas pela legislação.

Palavras-chave: Biossegurança, Equipamento de proteção individual, Clínicas odontológicas.

\begin{abstract}
This article aimed to verify the knowledge of dental students about biosafety standards. It was a descriptive study through the use of a questionnaire. The sample consisted of students completing the dental course $(n=30)$. Data were tabulated using the SPSS (Statistical Package for Social Sciences) program. $100 \%$ of the sample protects the patient; $68 \%$ use goggles; $96 \%$ discard sharps in suitable reservoir; $89 \%$ use rubber gloves to wash instruments; $96 \%$ use $70 \%$ alcohol as a disinfectant; $82 \%$ reported "good" knowledge of biosafety, and the existence of protocols for injured professionals; $83 \%$ avoid "touching objects with gloved hands"; and 68\% wash their hands before and after wearing gloves; $94 \%$ perform drill sterilization. Most academics are aware of biosafety protocols and perform clinical procedures in accordance with the standards set by law.
\end{abstract}

Keywords: Biosafety, Individual protection equipment, Dental clinics.

Resumen: Este artículo tuvo como objetivo verificar el conocimiento de los estudiantes de odontología sobre los estándares de bioseguridad. Fue un estudio descriptivo mediante el uso de un cuestionario. La muestra consistió en estudiantes que completaron el curso dental $(n=30)$. Los datos se tabularon utilizando el programa SPSS (Paquete Estadístico para Ciencias Sociales). El 100\% de la muestra protege al paciente; El 68\% usa gafas; El 96\% desecha objetos punzantes en un depósito adecuado; El $89 \%$ usa guantes de goma para lavar instrumentos; El 96\% usa alcohol al 70\% como desinfectante; El 82\% informó tener un "buen" conocimiento de bioseguridad y la existencia de protocolos para profesionales lesionados; $83 \%$ evita "tocar objetos con las manos enguantadas"; y $68 \%$ se lavan las manos antes y después de usar

${ }^{1}$ UNIFIP - Centro Universitário. Patos-Paraíba. *E-mail: tessiaborja@yahoo.com.br 
guantes; El 94\% realiza la esterilización por perforación. La mayoría de los académicos conocen los protocolos de bioseguridad y realizan procedimientos clínicos de acuerdo con los estándares establecidos por la ley.

Palabras clave: Bioseguridad, Equipo de protección individual, Clínicas odontológicas.

\section{INTRODUÇÃO}

A biossegurança, como ciência indispensável às áreas da saúde, compreende os protocolos e condutas técnico- científicas e educacionais, cuja adoção pelos profissionais da área de saúde ou afins, tem como objetivo evitar acidentes e contaminação cruzada em ambientes clínicos, ambulatoriais e/ou hospitalares (BRASIL, 1990; BRASIL, 1995; GONÇALVES ACS e TRAVASSOS DV,1996; BRASIL, 2006).

A equipe de saúde bucal, seja o cirurgião dentista, sejam os auxiliares, está sujeita à diversas formas de contaminação. Uma delas são aquelas provocadas por agentes biológicos patogênicos, que podem ser veiculados por fluidos orgânicos, através dos jatos emitidos por aerossóis, ou manipulados diretamente pelos profissionais, durante os atendimentos ou no processamento dos instrumentais. Esses agentes infecciosos são capazes de causar enfermidades diversas, através de infecções cruzadas. Por isso, tornase indispensável a adoção de medidas de biossegurança que minimizem esses efeitos danosos aos trabalhadores da odontologia (KRIEGER D, BUENO R e GARRIDO MCL, 2010).

A falta de cuidado de alguns profissionais com relação à biossegurança tem propiciado a ocorrência de infecção cruzada no consultório odontológico (SILVA PEB, PATROCíNIO MC e NEVES ACC, 2002). Considerando-se os níveis de prevalência das doenças nos países desenvolvidos, a cada sete dias o cirurgião-dentista atenderia, pelo menos dois pacientes com infecção herpética e um número desconhecido de pacientes HIV positivos. Os métodos para o controle da hepatite B (utilização de EPIs e processamento correto de materiais perfurocortantes) são válidos também para o controle da AIDS, bem como para as demais doenças (GALVANI LD et al., 2004). A infecção cruzada é um risco eminente na atividade clínica odontológica, sendo, portanto, indispensável que os profissionais adotem, rigorosamente, os protocolos de prevenção às doenças (TAIWO JO e ADERINOKUN GA, 2002; BRASIL, 2006; FREITAS RR, 2012), pois são essas condutas que serão responsáveis pela proteção da equipe de saúde bucal, dos pacientes, bem como dos ambientes de assistência odontológica, diminuindo o risco de transmissão de microrganismos e agentes causadores de doenças (FERREIRA RA, 1995; GONÇALVES ACS e TRAVASSOS DV, 1996; MEDEIROS UV et al.,1998; BRASIL 2000; GARBIN AJ et al., 2005; BRASIL, 2006).

As "Medidas de Precauções" são definidas como um conjunto de protocolos que controlam as infecções. Devem ser adotadas universalmente, como forma de diminuição dos riscos de transmissão de microrganismos nos serviços de saúde (SANTOS FS, et al., 2009). Essas precauções universais são: a) utilização de barreiras ou equipamentos de proteção individual; b) prevenção à exposições às secreções corporais e sangue; c) prevenção a acidentes com materiais perfurocortantes; c) manejo adequado nos casos de acidentes de trabalho que envolvam a exposição a sangue e fluidos orgânicos; d) manejo adequado durante procedimentos de descontaminação e manipulação de dejetos e resíduos nos serviços de saúde (SCHEIDT KLS, ROSA LBS e LIMA EF, 2006).

A utilização dos equipamentos de proteção individual (EPI) tem por objetivo impedir a infecção da equipe de saúde, bem como de pacientes, por microrganismos presentes em fluidos como sangue e secreções orofaríngeas. São considerados EPIs: luvas (de procedimentos ou cirúrgicas); gorro, avental impermeável, máscara e óculos de proteção (TEIXEIRA M e SANTOS MV, 1999).

Além disso, Rosa AC et al. (2006) enfatizaram a necessidade indispensável de uma assepsia rigorosa, do uso de antissépticos e da manutenção das imunizações em dia, para proteção individual de pacientes e equipe, bem como a utilização dos EPIs durante o atendimento odontológico. Também relataram que o preparo prévio da sala de odontologia, para realização dos procedimentos, é de suma importância, primando pelas medidas de higiene e limpeza adequadas, uso de desinfetantes, assim como a colocação 
de barreiras descartáveis recobrindo os equipamentos de uso frequentes nos atendimentos à pacientes. Ainda pode-se elencar o descarte dos materiais perfurocortantes em recipientes resistentes a ruptura, e dejetos biológicos em sacos adequados e identificados para coleta seletiva e descarte apropriado, jamais dispensados no lixo comum.

Frente ao exposto, o objetivo deste trabalho foi avaliar o conhecimento dos acadêmicos de odontologia de uma faculdade privada do sertão paraibano, a respeito do conhecimento das normas de biossegurança, bem como de suas aplicações na clínica odontológica.

\section{MÉTODOS}

Tratou-se de um estudo transversal, analítico e prospectivo, através de uma abordagem indireta, por meio de questionário, sem identificação do participante. Foram incluídos na pesquisa todos os alunos concluintes do curso de odontologia de uma faculdade privada, sendo o universo igual à amostra, totalizando 30 (trinta) discentes. Todos assinaram o Termo de Consentimento Livre e Esclarecido - TCLE, assentindo colaborar com a pesquisa. A coleta de dados foi realizada por meio da aplicação de um questionário estruturado com questões objetivas, baseado no estudo Engelmann Al, et al. (2010), através do qual se avaliou o conhecimento dos alunos referente às normas de biossegurança adotadas em clínica, como também ao uso correto dos Equipamentos de Proteção Individual (EPI) e às condutas tomadas na lavagem dos instrumentais (Figura 1).

O presente estudo seguiu os preceitos da bioética, baseado nas Resolução 466/2012, recebendo a aprovação do Comitê de Ética em Pesquisa das Faculdades Integradas de Patos (CEP/FIP), sob protocolo número: 2.012.155.

Figura 1 - Instrumento para Coleta de Dados.

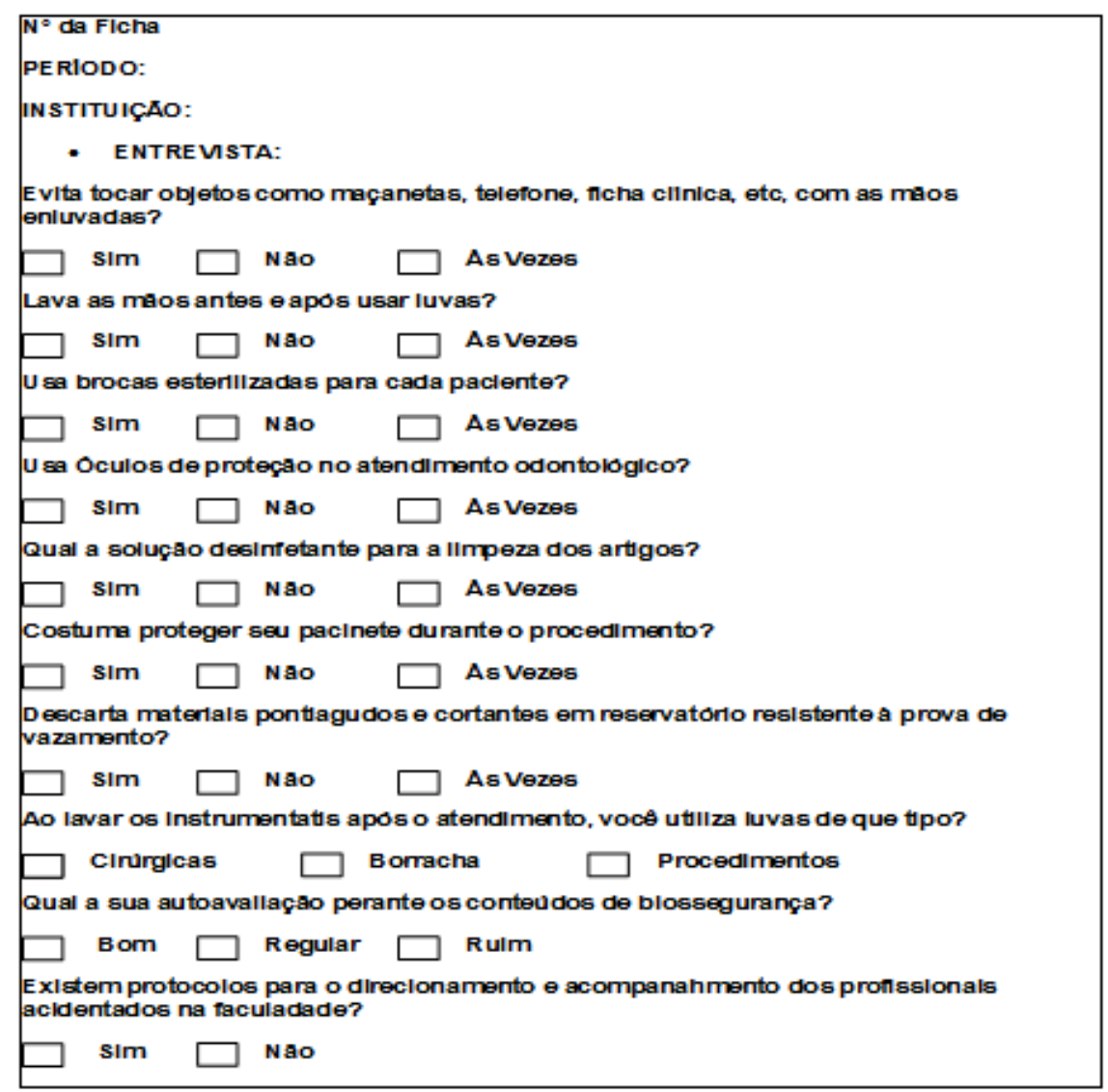

Fonte: Adaptado por Melo TRNB et al., 2019, baseado em ENGELMANN Al, et al., 2010. 


\section{RESULTADOS}

Os dados coletados foram tabulados e registrados no banco de dados do programa de informática SPSS (Statistical Package for Social Sciences) para Windows, versão 20.0, e descritos pelo programa Excel 2007 for Windows, sob forma de tabela.

Foram avaliados 30 questionários, conforme a descrição que consta no item métodos, de alunos concluintes do curso de odontologia. A média de idade foi de 24,5 anos e não houve divisão igualitária por sexo, sendo os participantes escolhidos de forma aleatória e espontânea.

De acordo com o método empregado no presente estudo, bem como as variáveis estudas, foi possível tabular dos dados de forma descritiva, abordando as variáveis estudadas: proteção ao paciente durante o atendimento; utilização de óculos de proteção; descarte de materiais perfurocortantes em reservatório resistente à prova de vazamento; utilização da luva de borracha para lavar o instrumental; tocar objetos com as mãos enluvadas; lavar as mãos antes e depois de usar luvas de procedimentos; utilização de substâncias desinfetantes e esterilização de brocas. Além disso, ainda se adotaram, como variáveis, a "Autoavaliação frente aos conhecimentos de Biossegurança" e a "Existência de protocolos para o direcionamento e acompanhamento de profissionais acidentados".

$\mathrm{Na}$ tabela abaixo, estão descritos os resultados referentes a todas as variáveis estudas, em números absolutos e relativos (percentualmente) (Tabela 1).

Tabela 1 - Distribuição da amostra de acordo com as variáveis estudas.

\begin{tabular}{lc}
\hline \multicolumn{1}{c}{ Variáveis } & Porcentagens \\
\hline Procedimentos de biossegurança & $100 \%$ \\
\hline "Proteção ao paciente durante o atendimento odontológico" & $68 \%$ \\
"Utilização dos óculos de proteção" & $96 \%$ \\
"Descarte de materiais perfurocortantes em reservatório & $89 \%$ \\
resistente à prova de vazamento" & $93 \%$ \\
"Utilização da luva de borracha para lavar o instrumental" & $68 \%$ \\
"Tocar objetos com as mãos enluvadas" & \\
"Lavar as mãos antes e depois de usar luvas de & $96 \%$ \\
procedimentos" & $4 \%$ \\
\hline Processamento de artigos & $94 \%$ \\
\hline "Solução desinfetante álcool à 70\%" & \\
"Solução desinfetante glutaraldeído" & \\
"Esterilização de brocas" & $82 \%$ (Bom Conhecimento) \\
\hline Protocolos de biossegurança & $82 \%$ (Existência de Protocolos) \\
\hline "Autoavaliação frente aos conhecimentos de Biossegurança" \\
"Existência de protocolos para o direcionamento e \\
acompanhamento de profissionais acidentados" \\
\hline
\end{tabular}

Fonte: Melo TRNB, et al., 2019.

\section{DISCUSSÃO}

Segundo Santos FS et al. (2009), a falta de cuidados básicos, ou mesmo a falta do conhecimento dada a estes cuidados por alguns profissionais contribui para a disseminação de doenças que poderiam ser evitadas de maneira simples. Apesar da sua importância, a prevenção da infecção cruzada se torna um grande desafio para todos, principalmente pela maneira como é negligenciada.

De acordo com os achados desse estudo, a maioria dos acadêmicos evita tocar objetos quando estão com as mãos enluvadas, o que demonstra cuidado e atenção na prevenção de infecções cruzadas, diminuindo o risco de contaminação para profissionais e pacientes. Esse dado corrobora com os relatos de Jorge AOC (2002) que, ao falar sobre os princípios de Biossegurança em Odontologia, enfatiza que as 
luvas devem ser usadas para a proteção do profissional e de seus pacientes; quando forem tocar em sangue, saliva, mucosas e tecidos; e até mesmo, num simples exame da cavidade bucal; sempre devem ser trocadas a cada atendimento odontológico.

Apesar de $1 / 3$ dos entrevistados não lavarem ou lavarem às vezes as mãos, antes e depois do uso das luvas, nota-se que a maioria dos acadêmicos, tem o hábito de realizar a higiene dos membros antes e após o uso do EPI, mostrando maior segurança no atendimento. Entretanto, esse dado vai de encontro ao preconizado por Brasil (2000), Brasil (2006) e Freitas RR (2012), e mostra-se como uma realidade preocupante, visto que a lavagem simples das mãos é responsável pela eliminação de quase todos os patógenos ali presentes, reduzindo drasticamente o risco de contaminação para pacientes e profissionais.

Quanto à utilização de brocas esterilizadas, observou-se que a maioria dos acadêmicos fazem uso desse recurso. Entretanto, por menor que seja a percentagem dos que violam os protocolos de biossegurança, é inaceitável a ocorrência desse fato, indo de encontro à literatura (TEIXEIRA M e SANTOS MV, 1999; BRASIL, 2000; JORGE AOC, 2002; BRASIL, 2006; FREITAS RR, 2012), visto que é inaceitável que profissionais da saúde negligenciem os protocolos de biossegurança, tendo em vista que o risco de infecção cruzada é altíssimo nos ambientes de saúde, e mais ainda, nos ambientes em que se prestam os serviços de atendimento odontológico.

No tocante ao uso dos óculos de proteção durante o atendimento, muitos dos entrevistados não fazem uso desse EPI, um número considerado alto, diante do risco a que está se expondo o profissional. Esse dado discorda da literatura, que preconiza o uso dos óculos, com proteções laterais, por todos os membros da equipe odontológica, em todo e qualquer procedimento (TEIXEIRA M e SANTOS MV, 1999; JORGE AOC, 2002; BRASIL, 2006).

Quanto ao tipo de solução desinfetante utilizada para a limpeza dos artigos de trabalho, o álcool a 70\% foi utilizado, seguido pelo glutaraldeído. Essa informação corrobora com os relatos de Jorge AOC (2002), que recomenda o álcool a $70 \%$ para desinfecção de nível intermediário, de artigos e superfícies; entretanto, vai de encontro ao que é preconizado em Brasil (2006), que recomenda o uso de gluteraldeído a $2 \%$, para desinfecção de artigos e superfícies.

O percentual de acadêmicos que utilizam as luvas de borracha para realizar a limpeza do instrumental após o atendimento foi relevante, corroborando com Brasil (2006), que considera indispensável, para proteção dos profissionais, o uso de luvas de borracha para todas as operações de higienização. Desta maneira, a maioria dos acadêmicos segue o recomendado pela legislação brasileira.

Para Jorge AOC (2002) as infecções que podem ocorrer no consultório são em tudo semelhantes às infecções hospitalares, que representam riscos aos pacientes em tratamento. Logo, a utilização de EPIs é indispensável. Em relação a esse protocolo, observou-se que todos os acadêmicos protegem os pacientes durante os procedimentos, corroborando com o preconizado pelo autor supracitado.

A maioria dos acadêmicos também descarta os materiais perfurocortantes em locais apropriados, o que consiste em um procedimento para diminuição dos riscos biológicos e de acidentes por parte dos funcionários que manipularão o lixo contaminado. Scheidt KLS, Rosa LRS e Lima EFA (2006) mencionaram que, nos ambientes em que são manipulados instrumentos perfurocortantes como vidros, fios de sutura, lâminas de bisturis, agulhas, fresas e brocas odontológicas, devem conter recipientes apropriados (resistentes a vazamentos e ruptura) para coleta desses materiais, assim como deverão estar próximos à realização dos procedimentos.

Grande parte dos acadêmicos entrevistados se auto avaliam "bons" perante seus conhecimentos sobre Biossegurança, ao passo que a minoria avaliaram seus conhecimentos como "regular". Segundo Santos FS et al. (2009) e Engelmann Al et al. (2010), a Biossegurança objetiva promover a eliminação e/ou diminuição a riscos minimamente baixos de contaminação nos procedimentos de tratamento de saúde e de pesquisas científicas. Nesse sentido, os cirurgiões dentistas, como profissionais que lidam diretamente com o risco eminente de infecção cruzada, necessitam, constantemente, de atualização dos conceitos referentes aos protocolos de biossegurança. 
Práticas de trabalho seguras envolvem a implementação e o desenvolvimento de uma política específica de revisão de procedimentos e alterações nas atividades realizadas pelos profissionais de saúde, de forma a reduzir a probabilidade de acidentes de trabalho envolvendo a exposição a materiais biológicos (BRASIL, 2006; SANTOS FS et al. (2009); ENGELMANN Al et al. (2010); FREITAS RR, 2012). Desta maneira, a implementação de protocolos para o acompanhamento dos profissionais que venham a se acidentar referese a um cuidado essencial a se ter nos estabelecimentos de atendimento. Essa informação corrobora com os dados descritos na presente pesquisa, em que a maioria dos entrevistados relataram que nas clínicas escolas da instituição, adota-se um protocolo para acidentes perfurocortantes, mostrando a preocupação com a biossegurança por parte dos gestores acadêmicos.

Grupos de acadêmicos de cursos de saúde são relatados na literatura como público alvo para adoção das condutas de biossegurança, visando o correto treinamento dos protocolos para minimizar e/ou eliminar por completo o risco de infecções cruzadas nos ambientes clínico - laboratoriais (FREITAS RR, 2012). Frente a isso, fica evidente a relevância de trabalhos que enfatizem esse tema, especialmente no tocante à amostra estudada.

\section{CONCLUSÃO}

De acordo com a metodologia empregada e os dados discutidos no presente trabalho, pode-se concluir que: a maioria dos acadêmicos executa os procedimentos clínicos de acordo com as normas de Biossegurança e utilizam os EPIs durante os atendimentos clínicos; as superfícies são desinfetadas com álcool à $70 \%$ em sua maioria, e todos os entrevistados relataram proteger os paciente durante os atendimentos. A maior parte dos avaliados descartam materiais perfurocortantes em reservatórios adequados e quase a totalidade dos acadêmicos se auto avaliam bons conhecedores do tema Biossegurança. A clínica escola da instituição estuada possui protocolos para direcionamento e acompanhamento dos profissionais acidentados.

\section{AGRADECIMENTOS}

Agradecemos a colaboração de todos os entrevistados e a todos que direta ou indiretamente contribuíram para a concretização desse estudo.

\section{REFERÊNCIAS}

1. BRASIL. Ministério da Saúde. Lei complementar no 791, de 9 de março de 1995. Estabelece o Código de Saúde no Estado. Diário Oficial do Estado, São Paulo, SP, $10 \quad$ mar. 1995. Disponívelem:<http://dobuscadireta.imprensaoficial.com.br/default.aspx?DataPublicacao=19950310\&Caderno=DOE I\& NumeroPagina=1>. Acesso em: 8 abril 2016.

2. BRASIL. Ministério da Saúde. Agência Nacional de Vigilância Sanitária. Serviços Odontológicos: Prevenção e Controle de Riscos. - Brasília: Ministério da Saúde, 2006.

3. BRASIL.Lei $\mathrm{n} \times 8080$, de 19 de setembro de 1990. Dispõe sobre as condições para a promoção, proteção e recuperação da saúde, a organização e o funcionamento dos serviços correspondentes e dá outras providências. Disponível em: <http://www.imesp.com.br> Acesso em: 08abril 2016.

4. BRASIL. Ministério da Saúde. Secretaria de Vigilância em Saúde. Programa Nacional de DST/Aids. Recomendações para atendimento e encaminhamento de exposição ocupacional a material biológico: HIV e Hepatites B e C. Brasília (DF): MS, 2000.

5. ENGELMANN Al, et al. Avaliação dos procedimentos realizados por cirurgiões-destistas da região de Cascavel-PR visando ao controle da biossegurança.Odontol. Clín.-Cient., Recife,2010;9(2)161-165.

6. FERREIRA RA. Barrando o invisível. Revista da APCD, 1995; 49(6):417-427.

7. FREITAS RR. Biossegurança em Odontologia. Monografia. Unversidade Federal de Minas Gerais. Curso de Especialização em Atenção Básica em Saúde da Família. Corinto, 2012.

8. GALVANI LR, et al. Utilização dos métodos de biossegurança nos consultórios odontológicos da cidade de Porto Alegre-RS. Stomatos. 2004 jan/jun; 10(18):7-13. 
9. GARBIN AJ, et al. Biosecurity in public and private office. J Appl Oral Sci. 2005;13(2):163-6.

10. GONÇALVES ACS, et al. Biossegurança do exercício da odontologia. RPG:Revista de Pós-Graduação, São Paulo, $1996 ; 3(3): 242-245$.

11. JORGE AOC. Princípios de biossegurança em odontologia.Rev. biociênc., Taubaté,2002; 8 (1):7-17.

12. KRIEGER D, et al. Perspectivas de biossegurança em odontologia. Rev Gestão Saúde. 2010;1(2):1-10.

13. MEDEIROS UV, et al. Uso das normas de controle de infecção na prática odontológica.RBO,1998; 55(1):209-215.

14. ROSA AC, et al. Control de la infección em odontologia parte 1 ra. Bol. Asoc. Argentina Odontol. Ninos, $2001 ; 30(1): 11-15$.

15. SANTOS FS, et al. Biossegurança: cuidados evitam riscos de infecção cruzada nos atendimentos odontológicos. Rev. da Associação Paulistana de Cirurgiões Dentistas. 2009; 63:117.

16. SCHEIDT KLS, et al. As ações de biossegurança implementadas pelas comissões de controle de infecções hospitalares. R Enferm UERJ, Rio de Janeiro, 2006 jul/set; 14(3):372-77

17. SILVA PEB, et al. Avaliação da conduta de biossegurança em clínicas odontológicas de graduação. Rev. biociênc., Taubaté, 2002; 8(1):45-52, jan.-jun.

18. TAIWO JO, ADERINOKUN GA. Assessing cross infection prevention measures at the Dental Clinic, University College Hospital, Ibadan.African Journal of Medicine and Medical Sciences, Oxford, 2002; 31(3):213-217.

19. TEIXEIRA M, SANTOS MV. Responsabilidade no controle de infecção. Rev. Assoc. Paulista CirurgiõesDentistas, 1999;53(3):177-189. 